\title{
Construction of the Tycho Reference Catalogue
}

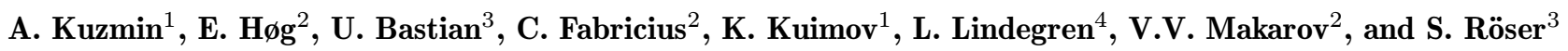 \\ 1 Sternberg Astronomical Institute, Universitetsky Prosp. 13, 119899 Moscow, Russia \\ 2 Copenhagen University Observatory, Juliane Maries Vej 30, DK-2100 Copenhagen Ø, Denmark \\ 3 Astronomisches Rechen-Institut, Mönchhofstrasse 12-14, D-69120 Heidelberg, Germany \\ ${ }^{4}$ Lund Observatory, Box 43, SE-22100 Lund, Sweden
}

Received November 20, 1998; accepted February 10, 1999

\begin{abstract}
The Tycho Reference Catalogue (TRC) project, as proposed in 1992 by the present authors, aimed to extend the Hipparcos reference system to fainter magnitudes. To assure rigidity of the reference system realization, the project relied on the Tycho Catalogue that provides highly-accurate positions of more than one million stars on the Hipparcos system. To maintain the precision of the reference system over longer periods of time, high-precision proper motions must be derived from Tycho and earlier ground-based measurements, most notably the century-old Astrographic Catalogue (AC).

The recently published first version of the Tycho Reference Catalogue provides highly-accurate positions and proper motions for 990182 stars of the Tycho Catalogue. It realizes the ICRS/Hipparcos system in the optical domain with a median accuracy of 45 mas at epoch J2000.0. The realization is based on stars of median magnitude $B_{\mathrm{T}}=11.25 \mathrm{mag}$, providing an average of 24 reference stars per square degree. The median accuracy of the TRC proper motions, estimated from external comparisons as $2.4 \mathrm{mas} / \mathrm{yr}$, ensures a reference system realization with an accuracy better than 100 mas over the time span of a few decades. Highly-accurate astrometry combined with homogeneous two-colour Tycho photometry makes TRC an ideal choice for a wide range of astronomical applications.
\end{abstract}

The present paper covers the major steps of the TRC construction, including the Astrographic Catalogue reduction onto the Hipparcos system, identification of the Tycho Catalogue stars in the AC, derivation of proper motions, a posteriori systematic corrections of the proper motions to account for residual AC systematic errors, and the TRC quality assessment. The relation of the TRC with the recently published ACT catalogue is outlined. A future version of the TRC, to be based on the new Tycho data

Send offprint requests to: A. Kuzmin

Correspondence to: avk@sai.msu.ru processing and a global block adjustment of the Astrographic Catalogue, is briefly discussed.

Key words: astrometry - reference systems catalogues — stars: kinematics

\section{Introduction}

At its 23rd General Assembly the International Astronomical Union (IAU) decided (Appenzeller 1998) to introduce from January 11998 the new International Celestial Reference System, ICRS (Arias et al. 1995). In accordance with the IAU 21st General Assembly Resolution on Reference Systems (Bergeron 1992), the ICRS is realized by extragalactic radio sources forming the International Celestial Reference Frame, ICRF (Ma \& Feissel 1997; Ma et al. 1998). ICRS and ICRF have thus replaced the previous reference system as realized by the FK5 fundamental catalogue.

The replacement of the FK5 system by the ICRS/ICRF has much deeper effects than a mere gain of precision. For the first time a reference system has been realized in the radio band rather than in the optical domain, which had been standard practice of astrometry for about a century. This decision was justified by the obvious advantages of using radio sources as reference objects, including higher random and systematic accuracy, long-time stability due to the lack of detectable proper motion, and independence of the equinox definition by means of the celestial equator and the ecliptic. In the optical domain, however, where the majority of all astronomical observations is still being performed, no comparable reference system realization existed.

The success of the European Space Agency (ESA) Hipparcos astrometric mission removed this deficiency. The Hipparcos mission yielded a catalogue of astrometric parameters of 118000 stars (ESA 1997), 
derived with milliarcsecond (mas) precision, and indirectly linked to ICRS with sub-milliarcsecond accuracy (Kovalevsky et al. 1997). Consequently, the IAU recommended the Hipparcos Catalogue as the primary realization of the ICRS in the optical domain.

However, for many applications, the ICRS realization by Hipparcos suffers from the excessive brightness of the reference stars (the median magnitude of the Hipparcos stars is close to $V=8.5 \mathrm{mag}$ ) and their low number density. Thus the primary reference system realization is practically inaccessible to many modern astronomical observations. This aggravates the problem of extending the reference system to faint stars, well-known to astrometry for decades. In the ICRS/Hipparcos context, that problem may be solved by the construction of a reference catalogue of many more fainter stars, which is directly linked to the Hipparcos system.

The most promising candidate for the purpose is the catalogue of more than 1000000 stars which resulted from the Tycho experiment on board the Hipparcos satellite (ESA 1997). The uniformly high systematic and random accuracy of the Tycho positions, its native link to the Hipparcos system, its relatively high star density and the availability of homogeneous two-colour photometry make the Tycho Catalogue an ideal means for extending the ICRS/Hipparcos frame to fainter stars.

The only drawback of Tycho as an astrometric reference catalogue is the poor quality of the proper motions. The proper motions of Tycho stars derived within the experiment have low precision, typically $30-40$ mas per year (mas/yr), which will deteriorate the reference frame within a decade.

The derivation of high-precision proper motions of Tycho stars as a way to construct a denser reference catalogue constituted the aim of the Tycho Reference Catalogue project, as proposed by the present authors in 1992 (Röser \& Høg 1993). The high-quality early-epoch observations necessary to derive proper motions with an accuracy of a few mas/yr, were to be provided by the Astrographic Catalogue (AC), a collection of twenty catalogues of position measurements at mean epoch near B1907.0, containing observations of circa 4.5 million stars brighter than $m_{\mathrm{pg}}=12 \mathrm{mag}$. Due to the early epoch and high intrinsic accuracy $(0$ '. $2-0$ '. 4$)$, AC constitutes an excellent first epoch for the massive derivation of proper motions.

The TRC pilot project (Kuzmin et al. 1997b), performed in 1996-97 using the early access to Hipparcos and Tycho data, confirmed that the $\mathrm{AC}$ makes it possible to derive proper motions with $2-3 \mathrm{mas} / \mathrm{yr}$ precision for nearly all Tycho stars. The pilot project also demonstrated the necessity to put AC onto the Hipparcos system and the crucial importance of this procedure for the quality of the resulting proper motions.

The publication of the Hipparcos and Tycho catalogues (ESA 1997) allowed full-scale realization of the
TRC project, resulting in the recently published Tycho Reference Catalogue which provides highly-accurate positions at epoch J2000.0 and proper motions of 990182 stars of the Tycho Catalogue. The TRC has been made available through astronomical data centres; the catalogue characteristics and derivation procedure were summarized in a recent paper (Høg et al. 1998a). In the present paper we describe various aspects of the TRC construction in detail, aiming to provide the catalogue user with an indepth knowledge of the observations and procedures used to derive the Tycho Reference Catalogue.

\section{Source Catalogues}

\subsection{The Astrographic Catalogue}

Astrographic Catalogue resulted from the international cooperation "Carte du Ciel" (CdC), initiated in 1887 in order to construct an all-sky photographic catalogue of all stars down to $m_{\mathrm{pg}}=11 \mathrm{mag}(\mathrm{AC})$, and a survey down to $m_{\mathrm{pg}}=14 \mathrm{mag}(\mathrm{CdC})$. Detailed description of the $\mathrm{AC}$ and CdC projects can be found elsewhere (Eichhorn 1974; Debarbat et al. 1988).

The Astrographic Catalogue provides rectangular coordinates as measured on the plate and magnitude estimates of about 8.6 million star images. The limiting magnitude of AC is roughly $m_{\mathrm{pg}}=12 \mathrm{mag}$, though some zones give coordinates of stars as faint as 13 mag. The basic information on the zonal catalogues comprising the $\mathrm{AC}$ is provided in Table 1 . It should be noted that by chance the plates centred at $+11^{\circ}$ were taken and measured twice, by the Bordeaux and Toulouse observatories. The Hyderabad Observatory was responsible for two zones. The Potsdam Observatory, originally responsible for the zone $+32^{\circ}$ to $+39^{\circ}$, had stopped the work soon after the First World War. Only about 30 per cent of the original plates taken and measured at Potsdam were published. Later on, this zone was reobserved at Oxford, Hyderabad and Uccle.

\subsubsection{Observation and measurement}

The Astrographic Catalogue was observed with so-called normal astrographs - a type of telescope built especially for the purpose, with an aperture of $33 \mathrm{~cm}$ and a focal length of $3438 \mathrm{~mm}$ (which gives a plate scale of $1^{\prime}=1 \mathrm{~mm}$ ). The photographs were taken on $16 \mathrm{~cm} \times 16 \mathrm{~cm}$ plates with a useable field of $13 \mathrm{~cm} \times 13 \mathrm{~cm}$, which is equivalent to $2.1^{\circ} \times 2.1^{\circ}$. Normal astrographs were used by all participating institutions except the Hyderabad Observatory (see Sect. 3.6.3).

Primary coordinate system on the AC plates was realized by the réseau - a silvered glass plate with a quadratic net of fine lines at $5 \mathrm{~mm}$ spacing, which was copied on every plate. The measurements of the positions of star images were made with respect to the photographic images 
Table 1. The zones of the Astrographic Catalogue

\begin{tabular}{|c|c|c|c|c|c|c|c|c|c|c|}
\hline \multirow[t]{2}{*}{ Observatory } & \multirow{2}{*}{\multicolumn{3}{|c|}{$\begin{array}{c}\text { Plate centres, } \\
\text { B1900.0 }\end{array}$}} & \multirow{3}{*}{$\begin{array}{r}\begin{array}{r}\text { Plates } \\
\text { taken }\end{array} \\
1149 \\
\end{array}$} & \multirow{3}{*}{$\begin{array}{r}\begin{array}{r}\text { Images } \\
\text { measured }\end{array} \\
392584\end{array}$} & \multirow{3}{*}{$\begin{array}{c}\begin{array}{c}\text { Mode of } \\
\text { measurement }\end{array} \\
\text { M, S }\end{array}$} & \multirow{2}{*}{$\begin{array}{l}\text { Coordinates } \\
\text { given to, }{ }^{\prime \prime}\end{array}$} & \multicolumn{3}{|c|}{ Plate epochs } \\
\hline & & & & & & & & Earliest & Latest & Mean \\
\hline Melbourne & $-90^{\circ}$ & to & $-65^{\circ}$ & & & & 0.060 & 1892.079 & 1940.482 & 1897.884 \\
\hline Sydney & $-64^{\circ}$ & to & $-52^{\circ}$ & 1400 & 743979 & $\mathrm{~S}$ & 0.300 & 1891.970 & 1948.208 & 1907.805 \\
\hline Cape & $-51^{\circ}$ & to & $-41^{\circ}$ & 1513 & 901229 & M & 0.060 & 1897.939 & 1911.988 & 1902.673 \\
\hline Perth & $-40^{\circ}$ & to & $-32^{\circ}$ & 1376 & 604315 & $\mathrm{~S}$ & 0.300 & 1902.390 & 1924.824 & 1911.499 \\
\hline Cordoba & $-31^{\circ}$ & to & $-24^{\circ}$ & 1360 & 467395 & $\mathrm{M}, \mathrm{S}$ & 0.300 & 1909.083 & 1915.712 & 1912.402 \\
\hline Hyderabad S. & $-23^{\circ}$ & to & $-17^{\circ}$ & 1260 & 521867 & S & 0.300 & 1914.935 & 1928.300 & 1918.995 \\
\hline Tacubaya & $-16^{\circ}$ & to & $-10^{\circ}$ & 1260 & 516609 & $\mathrm{~S}$ & 0.030 & 1900.076 & 1938.819 & 1904.450 \\
\hline San Fernando & $-9^{\circ}$ & to & $-3^{\circ}$ & 1260 & 346092 & M & 0.060 & 1891.760 & 1917.441 & 1897.206 \\
\hline Algiers & $-2^{\circ}$ & to & $+4^{\circ}$ & 1260 & 330449 & M & 0.006 & 1891.864 & 1911.883 & 1904.163 \\
\hline Toulouse & $+5^{\circ}$ & to & $+11^{\circ}$ & 1260 & 433083 & M & 0.006 & 1893.515 & 1935.506 & 1910.020 \\
\hline Bordeaux & $+11^{\circ}$ & to & $+17^{\circ}$ & 1260 & 355059 & M & 0.006 & 1893.031 & 1925.439 & 1905.419 \\
\hline Paris & $+18^{\circ}$ & to & $+24^{\circ}$ & 1261 & 436489 & M & 0.006 & 1891.727 & 1927.888 & 1895.670 \\
\hline Oxford & $+25^{\circ}$ & to & $+33^{\circ}$ & 1500 & 631791 & $\mathrm{~S}$ & 0.300 & 1892.683 & 1936.923 & 1908.065 \\
\hline Uccle & $+34^{\circ}$ & to & $+35^{\circ}$ & 320 & 158651 & M & 0.006 & 1942.426 & 1950.933 & 1944.948 \\
\hline Hyderabad N. & $+36^{\circ}$ & to & $+39^{\circ}$ & 592 & 242543 & $\mathrm{~S}$ & 0.300 & 1928.197 & 1937.662 & 1931.139 \\
\hline Helsingfors & $+40^{\circ}$ & to & $+46^{\circ}$ & 1008 & 284655 & M & 0.006 & 1892.639 & 1909.706 & 1895.087 \\
\hline Catania & $+47^{\circ}$ & to & $+54^{\circ}$ & 1008 & 319991 & M & 0.060 & 1894.660 & 1931.858 & 1903.235 \\
\hline Vatican & $+55^{\circ}$ & to & $+64^{\circ}$ & 1046 & 479975 & $\mathrm{E}$ & 0.300 & 1891.601 & 1926.192 & 1908.775 \\
\hline Greenwich & $+65^{\circ}$ & to & $+90^{\circ}$ & 1153 & 322133 & $\mathrm{~S}$ & 0.030 & 1892.253 & 1905.819 & 1896.989 \\
\hline Potsdam & $+32^{\circ}$ & to & $+39^{\circ}$ & 406 & 144001 & M & 0.030 & 1893.362 & 1900.151 & 1896.000 \\
\hline Totals & $-90^{\circ}$ & to & $+90^{\circ}$ & 22652 & 8632890 & & & 1891.601 & 1950.933 & 1907.621 \\
\hline
\end{tabular}

of the réseau lines. A secondary coordinate system was realized by a micrometer or a cross of scales in the eyepiece of the measuring microscope used to measure image coordinates relative to réseau lines. The mode of measurement employed by the individual observatories is given in Table 1; S means an eyepiece scale, $\mathrm{M}$ a micrometer screw, and $\mathrm{E}$ an eyepiece grid, a special measuring technique used by the Vatican Observatory only.

Brightness measures were derived by a number of methods: by measuring the image diameter, by matching the image with standard ones or by eye estimation (Gureeva 1992). The diversity of the AC brightness measures substantially impedes both their reduction to a modern magnitude system and the removal of the magnitude equation present in the measured coordinates. The worst cases in this sense are the Sydney, Cape and Perth zones due to the two distinct estimation methods used for every plate, one for the bright stars and another one for the faint stars. Consequently, the analysis of the magnitude equation in the data of these observatories was the most complicated of all. This situation could only be improved by using modern magnitudes for the stars.

\subsubsection{On the precision of published AC measurements}

The accuracy of the published measurements is defined by the measurement errors and the digitization errors. The latter is $D / 2 \sqrt{3}, D$ being the last decimal place of the published measurements. $D$ varies from observatory to observatory, ranging from 0 "'006 to 0 "' 3 (cf. Table 1 ). In the latter case the digitization error of 0 .'09 becomes comparable to the measurement and instrumental errors, estimated as $2 \mu \mathrm{m}$ or 0 .'12 each.

Furthermore, an analysis of the last digit of the published coordinates suggests that its distribution is far from uniform. In the Tacubaya zone, for example, only the figures $0,2,5,7$ or $0,3,5,8$ occur as the last digit, and on any given plate only one of these two sets is present. Other figures are rare, probably being misprints. Moreover, the last digit in those zones where it corresponds to $00^{\prime \prime} 3$ was found to be dominated by even numbers (or 0 and 5 , in case of the Vatican zone). A similar effect for the Hyderabad zone was previously reported by (Eichhorn \& Gatewood 1966). In such cases the digitization error is at least two times larger than the 0 .'09 value predicted from a uniform distribution of the last decimal place, reaching as much as 0 ". 43 in the case of the Vatican zone. Thus for at least half of all $\mathrm{AC}$ measurements (i.e. the zones where the measurements are given to $0 . \prime 3$ ) the accuracy of the published coordinates is defined by the digitization error rather than by the measurement accuracy. Obviously, a remeasurement of zones like Vatican or Tacubaya would nearly double the precision.

\subsubsection{Published data and the machine-readable version}

Publication of the $\mathrm{AC}$ measurements proceeded from 1902 to 1964 and resulted in 254 printed volumes of raw data. The machine-readable version of the Astrographic Catalogue was constructed at Sternberg Astronomical Institute (SAI) in 1987-94 (Nesterov et al. 1991; Gulyaev \& Nesterov 1992). Plate headers, running identification 

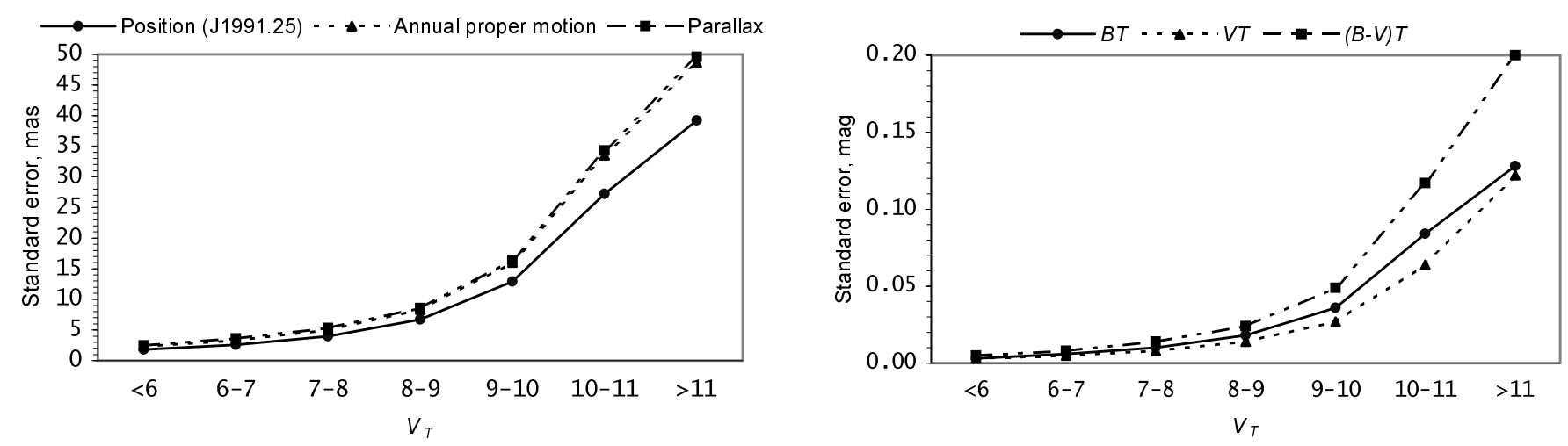

Fig. 1. Astrometric (left) and photometric (right) accuracy of the Tycho Catalogue, as a function of $V_{\mathrm{T}}$ magnitude (ESA 1997, Vol. 4)

number, rectangular plate coordinates and brightness measures were keypunched. All the misprints reported in published erratum lists were corrected in the data during keypunching. The machine-readable version of the Astrographic Catalogue includes all published measurements of the 19 completed zones plus measurements of 406 published plates of the original Potsdam zone.

Verification of the keypunched AC data included manual and automated procedures which assured that the data comply to the formats and record sequencing used in the published volumes, that all the fields are present and all the exceptions (incomplete or uncertain data) are marked with special flags. A substantial number of keypunching errors and 5-10 times more frequent unreported misprints were detected and corrected on the basis of an identification of the AC data with the HST Guide Star Catalogue (GSC, Lasker et al. 1990; Russell et al. 1990; Jenkner et al. 1990). The final error rate of the machinereadable $\mathrm{AC}$, i.e. of keypunching errors and misprints missing in the published errata lists and not found from AC-GSC matching, is estimated to be less than $0.1 \%$.

\subsection{The Tycho Catalogue}

The Tycho Catalogue is based on the observations with the star mapper on board the ESA Hipparcos astrometric satellite. A detailed description of the Tycho observational campaign, data processing and production of the catalogue can be found in the printed introduction (ESA 1997, Vol. 4).

\subsubsection{General characteristics}

The Tycho Catalogue provides positions at mean epoch J1991.25, proper motions, parallaxes and two-colour photometry (in $B_{\mathrm{T}}$ and $V_{\mathrm{T}}$ bands) of 1052031 stars brighter than $V_{\mathrm{T}}=11.5 \mathrm{mag}$. The Tycho astrometric data is referred to the ICRS system. The median standard error for stars at the median magnitude $V_{\mathrm{T}}=10.5 \mathrm{mag}$ and colour index $\left(B_{\mathrm{T}}-V_{\mathrm{T}}\right)=0.7$ mag is estimated as 25 mas in position and $0.06 \mathrm{mag}$ in photometry. Standard errors of Tycho astrometry and photometry as a function of $V_{\mathrm{T}}$ magnitude are shown in Fig. 1. The catalogue is more than 99 per cent complete down to $V_{\mathrm{T}} \sim 10 \mathrm{mag}$, the incompleteness basically occurring in dense fields.

\subsubsection{Tycho stars selection criteria}

The Tycho Catalogue is intended to be a complete sky survey, and therefore not all Tycho stars are plausible candidates for an astrometric reference catalogue like TRC. For the sake of reliability of the TRC stellar content, it was decided to exclude Tycho stars with unreliable astrometry or photometry, along with the high-proper-motion stars. The latter constraint was imposed in order to limit the number of misidentifications of the Tycho Catalogue stars in the AC (cf. Sect. 4.2). Specifically, the following entries of the Tycho Catalogue were rejected:

- Hipparcos stars not observed by Tycho;

- stars with positions derived from the Revised Tycho Input Catalogue;

- stars with standard error of $B_{\mathrm{T}}$ or $V_{\mathrm{T}}$ exceeding $0.3^{\mathrm{m}}$;

- stars with Tycho proper motion modulus exceeding $180 \mathrm{mas} / \mathrm{yr}$ at a $3 \sigma$ level.

The total number of Tycho stars surviving the rejection and consequently accepted for TRC was 1018531 .

\section{AC reduction onto the ICRS/Hipparcos system}

The Astrographic Catalogue reduction onto the modern reference system ICRS, as defined by the Hipparcos Catalogue, is of primary importance to any project aimed at the derivation of proper motions from the $\mathrm{AC}$ and modern-epoch observations. The TRC approach to this task (Kuimov et al. 1998) was basically the same as the one used for the AC reduction onto the FK5 system (Kuzmin et al. 1997a). This section outlines the approach, 
along with modifications necessitated by the change of reference system.

\subsection{Plate reduction strategy}

In general, the reduction model used to derive equatorial coordinates of stars from their measurements on a photographic plate can be described by the following relations:

$x=f\left(\alpha, \delta, m, c i, c_{1}, \ldots, c_{n}\right)$,

$y=g\left(\alpha, \delta, m, c i, c_{1}, \ldots, c_{n}\right)$.

The meaning of individual symbols in Eqs. (1) is as follows: $x$ and $y$ - image coordinates of the star as measured on the plate; $\alpha, \delta$ - equatorial coordinates of the star referred to some chosen reference system; $m, c i-$ its magnitude and colour; $f$ and $g$ - gnomonic projections supplemented by additional (usually polynomial) terms describing various plate-scale effects like tilt, coma etc.; $c_{1}, \ldots, c_{n}$ - the unknown parameters of the latter (socalled plate constants).

The plate reduction proper is the derivation of the unknowns $c_{1}, \ldots, c_{n}$ through Eqs. (1). Obtained plate constants then allow to solve Eqs. (1) for the equatorial coordinates $\alpha$ and $\delta$ of all stars measured on a plate or a set of plates.

Under the common approach to reduction, the socalled conventional plate adjustment, unknowns $c_{1}, \ldots, c_{n}$ are derived based on stars with known position at epoch of the plate exposure (i.e. reference stars). For the reduction to proceed, it is first necessary to investigate the significant plate-scale systematic errors or, in other words, to establish the functional form of $f$ and $g$ in Eqs. (1). Since neither normal astrographs nor measuring devices used throughout the AC project are anymore available for laboratory testing and calibration in their original state, the only method to investigate telescope-specific aberrations and measuring machine errors is to analyze the residuals after conventional plate adjustment. When the form of $f$ and $g$ - that is, the plate model - is established, a reduction of the $\mathrm{AC}$ data can proceed.

The establishment of the AC zone-specific plate models followed this approach. It started with a so-called Turner plate model, in which $f$ and $g$ are linear functions of the standard coordinates (rectangular coordinates on the plate derived from $\alpha, \delta$ through gnomonic projection with an assumed plate centre). After the least-squares solution of Eqs. (1) residuals from all plates of a zone were combined and analyzed for higher-order terms which significantly improve the model with respect to the chosen statistical criteria. The additional plate model terms defined in this way - the zone-specific corrections - may represent known physical phenomena (which is welcome), or be purely ad hoc (which cannot be entirely avoided).

To investigate significant plate-scale systematics in such a way, the number of reference stars per plate should be sufficient for a reliable determination of the parameters $c_{1}, \ldots, c_{n}$. However, the representation of the ICRS system by the Hipparcos Catalogue is too sparse for this purpose, providing an average 3 stars per square degree. Inapplicability of the Hipparcos for this purpose necessitated the employment of a denser intermediate reference catalogue and the development of a specific three-step plate adjustment procedure.

First, the plates constituting an AC zone were reduced on the "Astrographic Catalogue Reference Stars" (ACRS, Corbin \& Urban 1991) catalogue system by adjusting the 6 parameters of the Turner plate model. The residuals were then analyzed to yield additional terms. These then formed the advanced reduction model that accounts for the significant plate-scale systematic errors specific to the $\mathrm{AC}$ zone in question.

In step 2 the solution was repeated, but this time with the significant plate-scale terms found in step 1 added to the plate model. The ACRS again served as reference catalogue. The derived plate parameters were then analyzed for any dependence on observation/measurement conditions and other factors. Based on the results of this analysis, plates of the zone were, if necessary, discriminated into subsets. Subsequent averaging of the higherorder plate parameters yielded the final zone-specific (or subset-specific) systematic corrections.

In the third step the final reduction onto the Hipparcos system was performed using the Turner model and the Hipparcos Catalogue as reference catalogue. The zonespecific systematic corrections derived in step 2 were applied beforehand. If, for example, step 1 suggested that a sizeable cubic distortion is present for a specific astrograph, the distortion coefficient was estimated for all plates in step 2 . The estimates from individual plates were then averaged to yield a unique value, assumed to be characteristic for the astrograph. At step 3, that cubic distortion correction was then applied to all stars, as function of the actually measured plate coordinates.

The three-step approach using zone-specific corrections may be considered as an abridged plate overlap solution (Eichhorn 1960) in the sense that it assumes the instrument-specific parameters (e.g. the distortion coefficient) the same for all plates of the set, thus utilizing an important piece of information being lost under the individual plates adjustment approach. On the other hand, zone-specific plate models established under the present study provide the necessary basis for the future global block adjustment of the AC data.

\subsection{Intermediate reference catalogue}

The Hipparcos Catalogue cannot be used directly for the $\mathrm{AC}$ reduction due to its low star density: Hipparcos typically provides about 12 stars per $2.1^{\circ} \times 2.1^{\circ}$ AC plate 

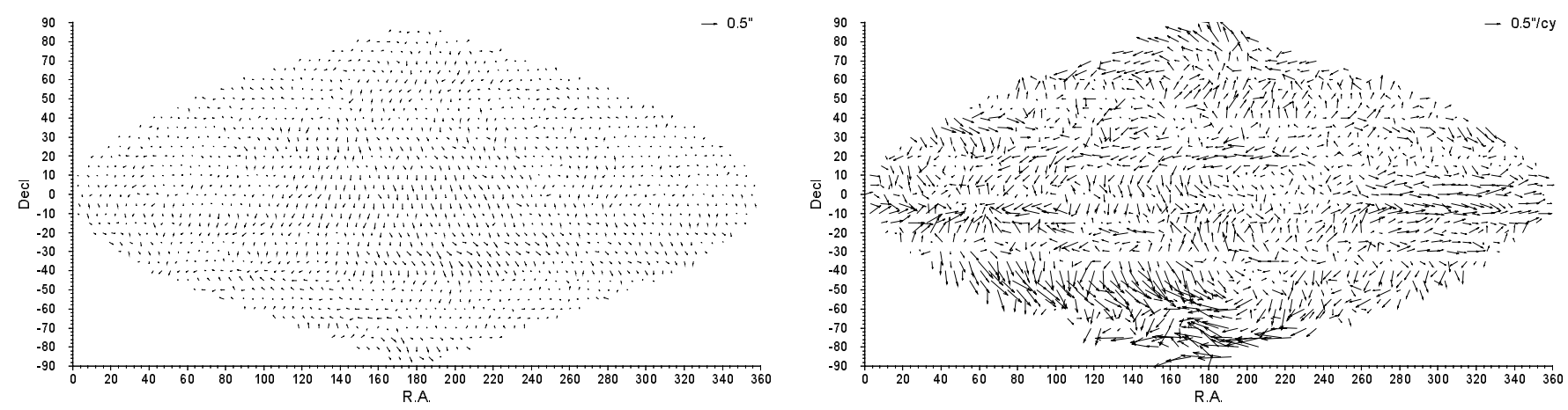

Fig. 2. Systematic differences ACRS-Hipparcos in position at mean epoch of the ACRS catalogue (left) and in proper motion (right). Mean differences averaged over cells of 25 square degrees are shown as two-dimensional vectors
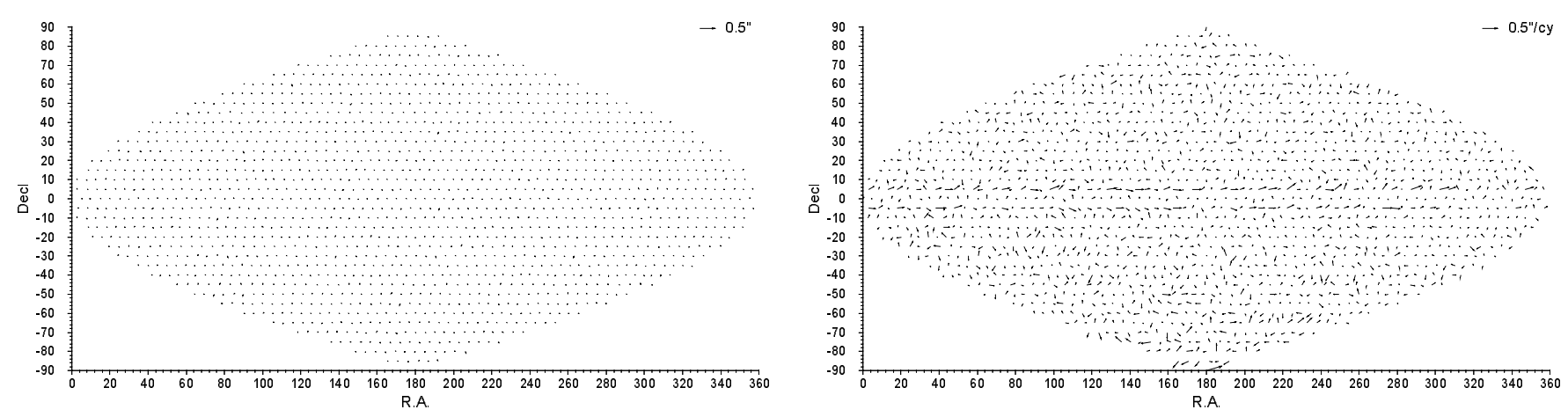

Fig. 3. ACRS-Hipparcos differences following the systematic corrections (compare with Fig. 2)

This number, though low, would be tolerable for the reduction with a linear model, provided the plate-scale systematic errors of the Astrographic Catalogue were known. Since the investigation of the plate-scale systematic errors present in the $\mathrm{AC}$ data is, in fact, one of the main subjects of the present study, an intermediate reference catalogue, tied to the Hipparcos system and providing at least 30-40 reference stars per AC plate (an equivalent of 10 stars per square degree), is necessary for the purpose.

At present, only two reference catalogues of the required density are available: "Astrographic Catalogue Reference Stars" (ACRS, Corbin \& Urban 1991), and "Positions and Proper Motions" (PPM, Röser \& Bastian 1991; Bastian et al. 1993), both utilizing practically all meridian and photographic observations of stars brighter than about $B=9.5 \mathrm{mag}$ available by 1990 . PPM is distinguished as the first major application of the Astrographic Catalogue for the derivation of proper motions. It should be noted that the AC reduction performed for PPM was limited to stars brighter than about $B=9.5 \mathrm{mag}$. Though it amounts only to about $10 \%$ of the Astrographic Catalogue stars, PPM is nonetheless correlated with $\mathrm{AC}$ and, consequently, cannot be applied for the investigation of systematic errors in AC. This leaves only ACRS as a suitable intermediate reference catalogue.

Prior to the application of ACRS to the investigation of systematic errors present in the AC data, it is necessary to remove the systematic errors of ACRS itself. Such errors were found in a comparison with Hipparcos during the TRC pilot project study (Kuzmin et al. 1997b). The errors amount to 250 mas in position at mean epoch and 5 mas/yr in proper motion (cf. Fig. 2). If unaccounted for, they would result in systematic errors of up to 500 mas when propagated to a typical AC epoch (the mean epoch difference between $\mathrm{ACRS}$ and $\mathrm{AC}$ is about 50 years).

Systematic patterns similar to the ones found in the ACRS-Hipparcos differences were previously reported from comparisons of FK5 and PPM with Hipparcos (Lindegren et al. 1995). This is not surprising: first, ACRS is on the FK5 system and, second, ACRS and PPM are based on nearly the same photographic material (apart from the AC data). This fact, along with the clear zonal structure of the systematic differences, makes it possible to attribute the latter mainly to systematic errors of the positional catalogues ACRS is based on.

Systematic differences of positions or proper motions given in any two catalogues may depend on position, magnitude and colour of the stars. The dependence on the latter two parameters, known as magnitude and colour equation respectively, has previously been difficult to investigate due to the absence of homogeneous set of magnitudes and colours for the large number of 
stars. Fortunately, the Tycho Catalogue photometry now provides the necessary basis for such an analysis.

Using the notation $\mu_{\alpha *}=\mu_{\alpha} \cos \delta$, we define systematic differences between ACRS and Hipparcos as

$$
\begin{aligned}
\Delta \alpha_{*} & =\left\langle\left(\alpha_{\mathrm{ACRS}}-\alpha_{\mathrm{HIP}}\right) \cos \delta_{\mathrm{HIP}}\right\rangle \\
\Delta \delta & =\left\langle\delta_{\mathrm{ACRS}}-\delta_{\mathrm{HIP}}\right\rangle \\
\Delta \mu_{\alpha *} & =\left\langle\mu_{\alpha * \mathrm{ACRS}}-\mu_{\alpha * \mathrm{HIP}}\right\rangle \\
\Delta \mu_{\delta} & =\left\langle\mu_{\delta \mathrm{ACRS}}-\mu_{\delta \mathrm{HIP}}\right\rangle
\end{aligned}
$$

where the angle brackets denote averages over suitable sets of stars (e.g. cells of 25 square degrees, as in Fig. 2).

The analysis of the ACRS-Hipparcos differences had shown that the main contribution has the form of a declination-dependent magnitude equation and, to lesser extent, a colour equation, to the first order given by:

$\Delta p=b_{0}+b_{1}\left(B_{\mathrm{T}}-9.15\right)+b_{2}\left[\left(B_{\mathrm{T}}-V_{\mathrm{T}}\right)-0.768\right]$.

Here $\Delta p$ denotes one of the systematic differences defined in Eqs. $(2), B_{\mathrm{T}}$ and $\left(B_{\mathrm{T}}-V_{\mathrm{T}}\right)$ are Tycho blue magnitude and colour, $9.15 \mathrm{mag}$ is the mean of $B_{\mathrm{T}}$ and $0.768 \mathrm{mag}$ the mean of $\left(B_{\mathrm{T}}-V_{\mathrm{T}}\right)$ of the stars common to ACRS and Hipparcos.

Figure 2 clearly identifies the zonal structure of the systematic differences between ACRS and Hipparcos positions and proper motions. Consequently, the coefficients $b_{0}, b_{1}$, and $b_{2}$ in (3) should depend on spherical coordinates, mainly on declination. To account rigorously for the latter, a fairly complex analytical or numerical model would be necessary. Moreover, the number of stars common to ACRS and Hipparcos is insufficient for the statistically reliable determination of the coefficients of an analytical expansion of the differences (e.g. by spherical harmonics). A reasonable approach to correct ACRS systematic errors would thus be to re-reduce the positional catalogues underlying ACRS. Since this time-consuming effort did not fit into the time schedule of the TRC project, a simple numerical method was used:

- A linear model of the type (3) was fitted to the ACRSHipparcos differences in position at mean ACRS epoch and in proper motion, both in $5^{\circ} \times 5^{\circ}$ cells, based on the residuals of 85794 astrometric standards of the Hipparcos catalogue identified in ACRS.

- Corrections to the position and proper motion of an individual ACRS star were derived by linear twodimensional interpolation of the corrections for the three closest cell centres, using Tycho magnitude and colour of the actual star.

The residuals after the systematic correction (Fig. 3) confirm that the major part of the ACRS systematic errors was removed. The residual systematic errors are below 100 mas in position at mean epoch and 1 mas/yr in proper motion. This improved version of the ACRS was used as an intermediate reference catalogue to derive the zonespecific corrections to the Astrographic Catalogue data.

\subsection{Identification of reference stars on $A C$ plates}

Prior to the plate reduction, it is necessary to identify the images of the reference catalogue stars on the AC plates. The problem is complicated by the difference between the coordinate systems which the measured rectangular coordinates and the standard coordinates are referred to. The two systems may differ by a displacement and a rotation, which for an AC plate may well amount to a few minutes of arc. Thus, to identify the reference stars among the measured images, at least an approximate transformation between the two coordinate systems is necessary. This could in principle be given by the provisional plate constants published along with the AC measurements. However, this approach would lead to a lot of additional efforts to transform the published constants for more than 22000 plates into machine-readable form. Furthermore, this would be unreliable due to typographical and keypunching errors.

To solve the problem in a consistent way, a patternmatching algorithm was developed (Kuimov 1992). It does not require a transformation between the rectangular coordinate systems of the source (reference stars) and target (measurements) to be known. The algorithm analyzes the triangles formed by stars of the source and target lists in search for pairs of similar triangles (similarity is tested by matching the respective angles, so the approach does not even require source and target coordinate system scales to be the same). The patternmatching algorithm was successful on all the 22652 Astrographic Catalogue plates, including all sorts of special cases.

\subsection{Reduction quality estimators}

As mentioned above, the establishment of an optimal reduction model for the plates of an Astrographic Catalogue zone was based on the analysis of the residuals after adjusting a linear plate model to AC data, by looking for significant additional plate-scale terms. This approach, close to step-wise regression, requires criteria according to which the optimal model is chosen. Since the aim of the $\mathrm{AC}$ reduction is to minimize the plate-scale systematic errors, it would be natural to consider that model as optimal which minimizes the so-called reduction error - a combination of the variance of the plate parameters and the residuals resulting from the incompleteness of the plate model. To estimate the reduction error, it is common practice to use criteria based on the (properly normalized) rms scatter of the residuals. For example, under the step-wise regression approach the optimal model is usually chosen on the basis of a Fisher test applied to the rms scatters of the successive models.

The residual scatter of a conventional plate adjustment represents four main factors: the variance of the adopted plate parameters, systematic plate-scale effects unmodelled by the adopted plate model, random measurement 
errors, and the random errors of the reference star positions at the epoch of observation. Due to the early epoch of the Astrographic Catalogue, the reference catalogue's positional accuracy at the epoch of the plates is comparable to the measurement errors (e.g., about 200 mas for Hipparcos stars). Consequently, these two components dominate the rms scatter, which under such circumstances becomes a fairly insensitive estimate of the reduction quality. Another disadvantage of the residual scatter as a reduction quality estimate is that it is limited to the magnitude interval of the reference stars. Thus its application as an estimate for other, usually fainter, stars is a mere extrapolation.

Fortunately, the two-fold overlap of the Astrographic Catalogue plates provides a natural estimator of the reduction quality - the variance of positions of the same star derived from overlapping plates. To avoid a bias introduced by proper motions, the estimate must be based only on plates with insignificant epoch difference (less than, say, 5 years). Consider a subset of AC plates containing $N_{\mathrm{S}}$ stars with at least two measurements. Let $N_{\mathrm{O}}$ denote the total number of measurements, $S_{i}$ the dispersion of the individual positions of $\operatorname{star} i, 1 \leq i \leq N_{\mathrm{S}}$, and $S$ the sum of these dispersions over all stars. Then the mean error of an individual position $s$ and its standard deviation $\sigma(s)$ can be estimated as follows (Hudson 1964)

$s^{2}=\frac{S}{N_{\mathrm{O}}-N_{\mathrm{S}}}, \sigma^{2}(s)=\frac{s^{2}}{2\left(N_{\mathrm{O}}-N_{\mathrm{S}}\right)}$.

The quantities $s_{\alpha *}$ and $s_{\delta}$, given by Eqs. (4) for the variance in right ascension and declination respectively, were used as a reduction quality estimates. The error of an individual star position includes a random part (measurement error) and a systematic part (reduction error). Since the former is not expected to vary significantly if measurements of the same star on overlapping plates are considered, smaller $s_{\alpha *}$ and $s_{\delta}$ values should correspond to smaller systematic errors. Moreover, due to the complete overlap, the estimates are based on all AC stars observed at least twice, and thus they solve the accuracy estimation problem for faint stars. Finally, the estimates are calculated from measurements of the same star, not from the residuals between measurements and reference star positions, and thus are not directly influenced by the errors of the reference catalogue.

These reasons make the variance of positions from overlapping plates suitable for the assessment of the reduction quality, both for the establishment of the optimal reduction model and for the estimation of the accuracy of an individual star positions after the final reduction step (cf. Sect. 3.7).

\subsection{The standard reduction model}

Analysis of the residuals after the first step of the reduction procedure suggested the following plate model to be optimal with respect to the minimum systematic errors criterion:

$$
\begin{aligned}
& f=\frac{c_{1} \xi_{1}+c_{2} \eta_{1}+c_{3}}{1+c_{7} \xi_{1}+c_{8} \eta_{1}}+c_{10} x_{1}+c_{11} y_{1}+c_{14} b x+c_{15} b \\
& g=\frac{c_{4} \xi_{1}+c_{5} \eta_{1}+c_{6}}{1+c_{7} \xi_{1}+c_{8} \eta_{1}}+c_{12} x_{1}+c_{13} y_{1}+c_{16} b y+c_{17} b
\end{aligned}
$$

The meaning of individual symbols in Eqs. (5) is as follows: $c_{1}, \ldots, c_{17}-$ unknown plate parameters; $x$ and $y-$ measured coordinates of a star image in units of $\mathrm{mm} ; b-$ measure of the star's brightness; $\xi$ and $\eta$-standard coordinates; $\xi_{1}=\xi+c_{9} \xi\left(\xi^{2}+\eta^{2}\right)$ and $\eta_{1}=\eta+c_{9} \eta\left(\xi^{2}+\eta^{2}\right)$ - distorted standard coordinates. The terms with $x_{1}=$ $(x+70) \bmod p$ and $y_{1}=(y+70) \bmod p$ are included to account for the systematic errors of the measuring device (see Sect. 2.1.1); $p$ stands for the réseau interval (typically $5 \mathrm{~mm}$, with the only exception of Vatican zone where due to the use of a very special measuring technique $p$ should be set to $10 \mathrm{~mm}$ ).

The standard coordinates $\xi$ and $\eta$ in Eqs. (5) were computed from the apparent positions of the reference stars. This approach ensures that differential refraction and annual aberration are taken into account prior to plate reduction, so that the plate parameters $c_{7}, \ldots, c_{17}$ of Eqs. (5) characterize exclusively the astrograph and measuring device errors.

\subsection{Reduction of specific $A C$ zones}

To illustrate the reduction approach outlined above, the next few sections cover the reduction of three AC zones: Melbourne, Hyderabad, and Algiers. Of these, the Melbourne zone is described in most detail; for the other two the emphasis is put on the zone-specific features.

\subsubsection{The Melbourne zone}

The Melbourne Observatory treated the zone $-90^{\circ}$ to $-65^{\circ}$ (declination limits of $\mathrm{AC}$ zones are referred to the equinox B1900.0). A description of the observation and measurement procedures can be found in the introduction to the published data (Baldwin 1926). A total of 1149 plates were taken between 1892 and 1940, the mean plate epoch being J1897.9.

The instrument used for taking the plates was a normal astrograph made by "Grubb". A total of 392584 images were measured with the aid of six different measuring devices, some of micrometer screw and some of eyepiece scale type. The measured coordinates are given to $1 \mu \mathrm{m}(0 . \prime 06)$; the distribution of the digits in the last decimal place is close to uniform. The brightness estimates are given in the form of measured image diameters for bright stars and eye estimates for faint ones.

The residuals after the first step of the reduction procedure (using ACRS as the reference catalogue and a linear plate model) are shown in Figs. 4a and b. Two type of 

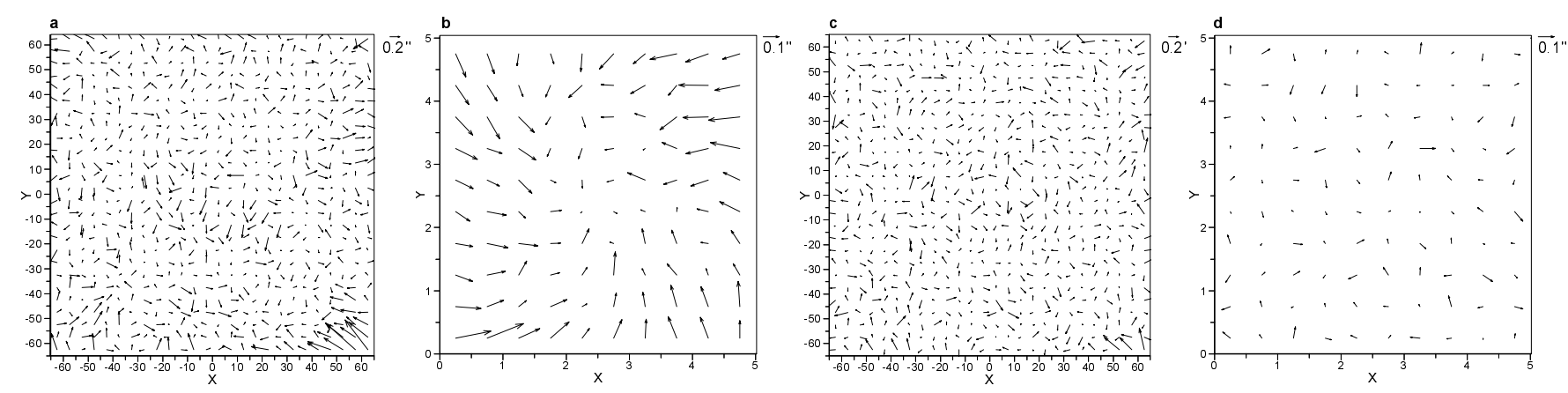

Fig. 4. Systematic differences between AC Melbourne and the reference catalogue. Residuals from 1149 plates were averaged in $5 \times 5 \mathrm{~mm}$ cells over the plate (plots a and c), and in $0.5 \times 0.5 \mathrm{~mm}$ cells over the measuring microscope field b) and d). a) and b): residuals after the first step of the reduction procedure, with the AC data reduced onto the ACRS system by linear plate model; c) and d): residuals after the systematic correction and the final reduction onto the Hipparcos system
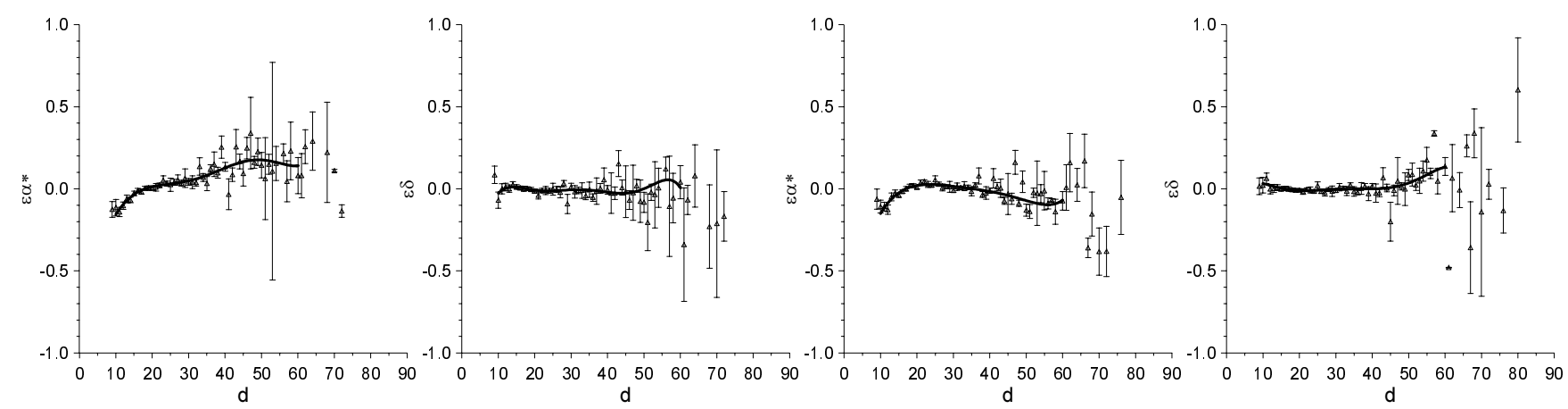

Fig. 5. AC Melbourne residuals in right ascension $\left(\epsilon_{\alpha *}\right)$ and declination $\left(\epsilon_{\delta}\right)$, in units of arcseconds, as functions of the brightness measure (measured image diameter). Left: AC Melbourne-ACRS residuals before magnitude equation correction; right: AC Melbourne-Hipparcos after the correction. The curves represent 6th order polynomial fits to the residuals

plots illustrate the plate-scale systematic errors and the errors of the secondary coordinate system, respectively.

Figure 5 shows the residuals in right ascension and declination as functions of measured image diameter, with the magnitude equation in right ascension clearly indicated. It is nonlinear and reaches 0 "! 3 for faint stars. A magnitude equation of this type cannot be attributed to ordinary aberrations of the optical system, nor to the atmospheric dispersion (plates were taken close to the meridian, so the latter may cause a magnitude equation in declination only). The most probable cause is a defect of the objective lens, e.g. a decentering, or imperfect guiding.

The second step of the reduction procedure yielded 1146 collections of plate parameters $c_{1}, \ldots, c_{17}$. Three plates were excluded from the derivation of the zonespecific corrections due to an insufficient number of reference stars. The plate parameters $c_{7}, \ldots, c_{17}$ were subsequently averaged to yield coefficients of the zone-specific corrections; the parameters $c_{10}, \ldots, c_{13}$, describing the secondary coordinate system errors, were averaged for each measuring device.

In the third step, the final reduction on the Hipparcos system was performed using the Turner model and the Hipparcos Catalogue as reference. Corrections for the zone-specific systematic errors derived in step 2 were applied beforehand. The residuals after the final reduction are shown in Figs. 4-5.

\subsubsection{The Algiers zone}

The Algiers Observatory treated the zone $-2^{\circ}$ to $+4^{\circ}$ of declination. Description of the observation and measurement procedures can be found in the introduction to the published data (Trepied 1903). A total of 1260 plates were taken between 1891 and 1912, the mean plate epoch being J1904.2.

The plates were taken with the normal astrograph manufactured by "Henry Brothers". Coordinates of the 330449 images were measured with the aid of micrometer screw devices, the usual practice for the French AC zones. The measured coordinates are given to $0.1 \mu \mathrm{m}\left(00^{\prime \prime} 006\right)$. Magnitudes derived from the measured image diameters are given for all stars.

The Algiers observations were found to be among the best with respect to both random and systematic errors. Both plate-scale and measuring machine systematic errors are small and could be corrected very well. The overall magnitude equation in right ascension is close to zero, except for plates taken between 1900 and 1905 (Fig. 6). 


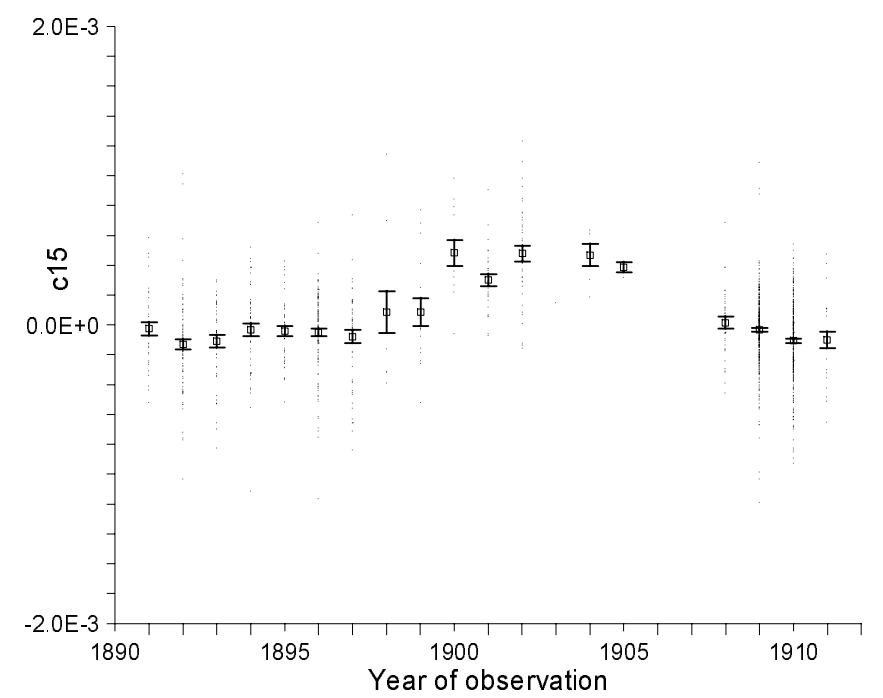

Fig. 6. The plate parameter $c_{15}$, characterizing the magnitude equation in right ascension, given for the AC Algiers plates as function of the year of observation

However, a subdivision of the plates into two sets according to the exposure date did not improve the overall reduction quality as estimated by the position variance criterion. Therefore it was rejected. It should be noted that the same feature was detected in the AC Bordeaux and Oxford data. In the latter case, the change in the magnitude equation may be associated with the adoption of a new emulsion. Unfortunately, the French observatories did not document the emulsion type being used.

\subsubsection{The Hyderabad zone}

The Nizamiah Observatory, located at Hyderabad, India, actually observed two zones: $-23^{\circ}$ to $-17^{\circ}$ (referred to as Hyderabad South), and $+36^{\circ}$ to $+39^{\circ}$ (Hyderabad North). The reduction of the southern zone is described in the following, though the results apply to the northern one as well.

A description of the observation and measurement procedures can be found in the introduction to the published data (Pocock 1918). A total of 1260 plates for the Hyderabad South zone were taken between 1914 and 1929, the mean plate epoch being J1919.0. The coordinates of 521867 images were measured with an eyepiece scale device.

The Nizamiah Observatory was the only one of the participating institutions to use a non-standard objective, probably the so-called "Cook Triplet", with the focal length of $3.37 \mathrm{~m}$. The cubic distortion coefficient $c_{9}$ of the Hyderabad telescope was found to depend on the air temperature at exposure time (cf. Fig. 7). The dependence was approximated by a one-year sinusoid, as shown in Fig. 7, to account for the effect. No such feature has been found in any other $\mathrm{AC}$ zone.

\subsection{Overview of the reduction results}

The plate model given by Eqs. (5) and, for some AC zones, the shortcut without magnitude-dependent terms, yielded the minimum values of the reduction quality estimates $s_{\alpha *}$ and $s_{\delta}$ among all reasonable models tried. Consequently, the AC reduction onto the Hipparcos system relied on this plate model, considered as optimal with respect to the reduction quality estimates. Table 2 lists, for all zones of the Astrographic Catalogue, the reduction quality estimates along with rms scatter of the residuals (in units of mas), both after the first step of the reduction procedure (reduction onto the ACRS system by a linear model without systematic corrections) and after the final reduction onto the Hipparcos system, with zone-specific systematic corrections applied to the $\mathrm{AC}$ data.

The differentiation between plate models with and without magnitude-dependent terms is due to the fact that the magnitude equation is by far the most complicated type of systematic error found in the AC data. A noteworthy example of the non-linearity and complexity of the magnitude equation is the one detected in the Melbourne zone (cf. Fig. 5). Also a colour equation, though less pronounced, was found in the AC data.

Our approach to the treatment of the magnitude equation was a compromise between the statistical uncertainty of its determination by the conventional plate adjustment technique and the necessity to eliminate the effect, at least in the most prominent cases. The decision as to which type of plate model given by Eqs. (5) - with or without

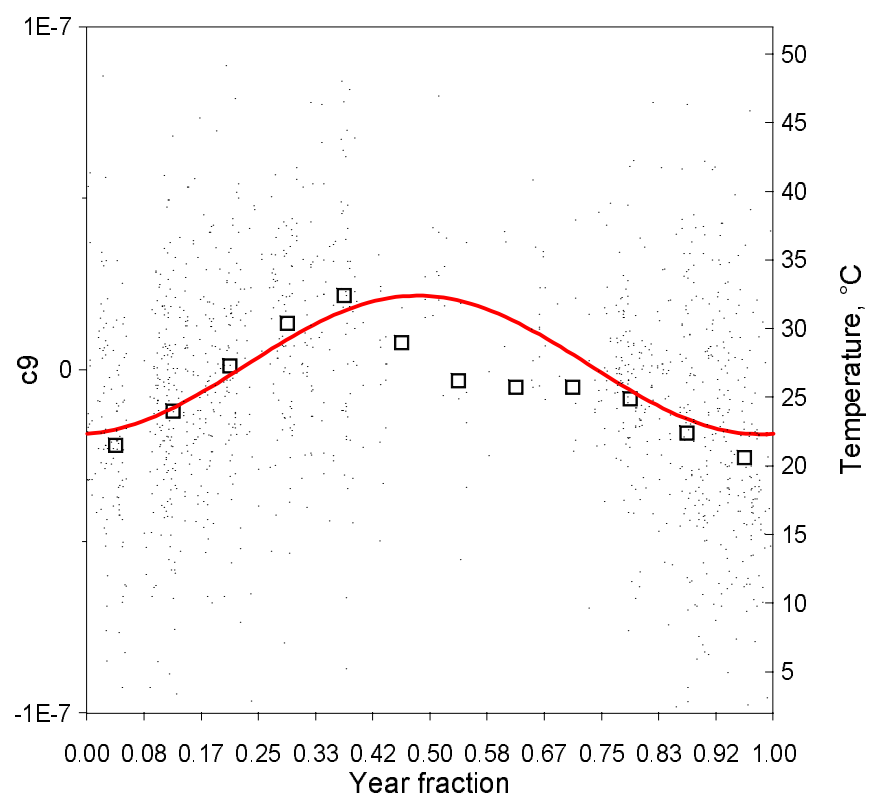

Fig. 7. The cubic distortion coefficient of the Hyderabad Observatory telescope (dots, referred to the left-hand axis) and monthly mean air temperature in Hyderabad (boxes, referred to the right-hand axis), as functions of season. The solid line is described in the text 
magnitude-dependent terms - should be adopted for each $\mathrm{AC}$ zone was made on the basis of two criteria: the size of the magnitude equation from the $\mathrm{AC}-\mathrm{Hipparcos}$ residuals, and the comparison of the reduction quality estimates of the two plate models. It should be noted that the two criteria estimate the magnitude equation in distinct magnitude intervals: the comparison with Hipparcos provides information on bright stars, while the reduction quality estimates, being dominated by faint stars, estimate the magnitude equation at the faint end. If the introduction of the magnitude-dependent terms increased the reduction quality, or if a sizeable magnitude equation was found from the comparison with Hipparcos, the magnitude-dependent terms were adopted. The AC zones reduced by plate model (5) with the magnitude-dependent terms are checked in the last column of Table 2 .

As can be seen (cf. Table 2), the values for the reduction quality estimates after the first step of the reduction procedure are always lower than those after the third step. This is due to the higher density of the ACRS Catalogue, which leads to smaller variances of the plate parameters and, thus, to smaller values of the reduction quality estimates. On the other hand, the better systematic quality of the reduction onto the

Table 2. Reduction quality estimates and rms scatter of the residuals (in units of mas) after the first step of the reduction procedure (ACRS as reference catalogue, Turner model, no systematic corrections) and after the third step (Hipparcos as reference catalogue, Turner model, zone-specific systematic corrections applied). The last column denotes whether the final plate model had magnitude-dependent terms added

\begin{tabular}{lccccccc}
\hline Observatory & \multicolumn{3}{c}{ After 1st step } & \multicolumn{3}{c}{ After 3rd step } & ME \\
& $s_{\alpha *}$ & $s_{\delta}$ & $\sigma$ & $s_{\alpha *}$ & $s_{\delta}$ & $\sigma$ & \\
\hline Melbourne & 439.0 & 412.2 & 605.3 & 444.9 & 416.6 & 367.5 & + \\
Sydney & 539.4 & 479.0 & 625.5 & 571.7 & 508.3 & 520.1 & + \\
Cape & 471.8 & 461.1 & 571.4 & 499.1 & 485.3 & 439.3 & + \\
Perth & 464.6 & 439.5 & 601.7 & 478.0 & 468.0 & 389.8 & \\
Cordoba & 451.6 & 396.5 & 563.0 & 532.8 & 499.2 & 416.3 & + \\
Hyderabad S. & 597.4 & 559.5 & 446.3 & 526.6 & 524.2 & 327.9 & + \\
Tacubaya & 355.7 & 337.1 & 451.4 & 439.4 & 419.7 & 357.8 & \\
San Fernando & 432.1 & 460.4 & 459.7 & 546.9 & 534.5 & 345.1 & + \\
Algiers & 249.7 & 241.1 & 380.6 & 403.6 & 378.6 & 281.8 & + \\
Toulouse & 353.3 & 310.4 & 400.5 & 445.8 & 403.1 & 327.8 & \\
Bordeaux & 288.6 & 280.3 & 378.8 & 354.4 & 340.1 & 316.1 & \\
Paris & 333.4 & 307.8 & 392.1 & 396.8 & 367.5 & 311.5 & \\
Oxford & 420.8 & 394.1 & 448.7 & 506.4 & 482.2 & 352.7 & + \\
Uccle & 565.7 & 538.5 & 567.6 & 509.7 & 510.6 & 352.7 & + \\
Hyderabad N. & 601.1 & 545.9 & 428.6 & 458.9 & 441.7 & 313.4 & + \\
Helsingfors & 341.3 & 318.6 & 467.6 & 389.0 & 374.0 & 314.8 & \\
Catania & 451.1 & 425.3 & 469.9 & 484.7 & 470.0 & 318.0 & + \\
Vatican & 495.9 & 495.6 & 551.7 & 524.4 & 535.7 & 543.3 & \\
Greenwich & 368.2 & 371.9 & 478.7 & 427.3 & 434.6 & 359.5 & \\
Potsdam & 350.7 & 328.4 & 450.1 & 393.2 & 380.9 & 397.8 & \\
\hline Mean values & 428.6 & 405.2 & 487.0 & 466.7 & 448.7 & 367.7 & \\
\hline
\end{tabular}

ACRS system (as indicated by the smaller values for the reduction quality estimates) is limited to the scale of an AC plate, while on larger scales the systematic errors of the ACRS still persist. Using Hipparcos at the final step of the reduction ensures better systematic quality at the global scale, which is most important for many applications. However, it is achieved at the price of larger systematic errors on the scale of about one degree.

The reduction quality estimates and the rms scatter of the residuals represent two different attempts to assess the accuracy of the derived star positions. In the ideal case of an error-free reference catalogue and equal measurement errors of all stars, the two should coincide. In the real world, the reduction quality estimates may exceed the rms scatter or vise versa, depending on the actual balance between the reference catalogue errors, the plate model completeness and the presence of magnitude equation. The dominant role in both estimates is played by the random errors of the $\mathrm{AC}$ observations. However, the rms scatter of the residuals additionally reflects the reference catalogue errors. Accordingly, Table 2 shows that rms scatter with respect to Hipparcos is 1.5 times less than that with respect to ACRS. This is partly due to the superior quality of the Hipparcos proper motions, and partly to the higher correlation of the reduced AC positions of Hipparcos stars with the Hipparcos data. The latter is caused by the significantly smaller number of reference stars per plate.

Reduction quality estimates are based on all stars with two or more observations, and thus these estimates of the accuracy of the reduced AC measurements refer to faint stars. A test computation of $s_{\alpha *}$ and $s_{\delta}$ using only Tycho stars (which on average are about one magnitude brighter) resulted in values which are about 30 per cent smaller than those given in Table 2 .

\section{Identification of Tycho stars in AC}

Once the individual positions are brought to the same reference system (namely, ICRS/Hipparcos), the only step left before the derivation of proper motions is to collect those that belong to the same star, i.e. to identify Tycho stars in the Astrographic Catalogue. Prior to describing the actual identification procedure, we should emphasize the importance of this step for the construction of a reference catalogue. The overall reliability of the catalogue contents, and especially of the proper motions, to a great extent depends on the correctness of this procedure.

\subsection{Identification by position}

The identification of observations is significantly different from the reference stars identification problem addressed in Sect. 3.3, the two procedures having different objectives: 
the former aims to establish a definite correspondence between individual observations of the same object, while for the latter such a correspondence is merely an intermediate step necessary to adjust the coordinate system of the plate measurements to that of the reference catalogue.

The standard procedure of identification by position, the so-called window-based approach, defines a window, i.e. a cone of some predefined size centred on the object to be identified (in our case, the Tycho star). It then studies the candidates, i.e. observations (in our case, the images measured on the AC plates) lying within the window. The most suitable candidate is then used to assign the identification.

Obviously, the absence of robust characteristics, like e.g. the pattern parameters of the reference stars identification procedure, makes the window-based approach highly sensitive to errors and peculiarities of the individual observations. The random and systematic errors of position as well as duplicity or variability may lead to a misidentification, which in the TRC case will result in a large error of the derived proper motion.

\subsection{Additional constraints}

To lower the number of probable misidentifications and thus to assure reliability of the TRC contents, the standard window-based approach was complemented by constraints on Tycho stars and Astrographic Catalogue observations.

1. Rejection of stars with high Tycho proper motion: identification of all Tycho stars without limiting the proper motion would result in the necessity of a large window, which in turn would lead to an intolerably high number of misidentifications. To avoid this, only Tycho stars with a modulus of the Tycho proper motion less than 180 mas/yr were subjected to identification. The threshold value corresponds to the lower limit of the proper motion modulus of stars included in the NLTT catalogue (Luyten 1979; Luyten \& Hughes 1980).

As a result, stars with high proper motion are missing in TRC. This may be a drawback from the point of view of stellar kinematics, but proper motions of the stars in question are generally available from Hipparcos.

2. Dynamic window: the window size was adjusted individually for every $\mathrm{AC}$ plate by the limit on the proper motion modulus and the difference between plate epoch and mean epoch of the Tycho Catalogue, J1991.25.

3. Isolation of observations subject to identification: an identification was accepted as such only if both the Tycho star and the measured image on the particular AC plate were the only representative of the respective catalogue within the identification window.
This constraint was necessitated by the absence of suitable photometric data in the Astrographic Catalogue, which prevented isolation of observations on the basis of brightness.

The window-based approach with the constraints outlined above allowed one-to-one identification for $96 \%$ of the Tycho stars satisfying the selection criteria (cf. Sect. 2.2.2). Reliable identification for an additional $2 \%$ of the Tycho stars was obtained by the special treatment of the problem cases.

\subsection{Problem cases}

Two types of problem cases were handled:

- "Field star": a Tycho star with two AC candidates within the identification window, one of them usually being an image of a fainter, unrelated (field) star. The problem cases of this type were solved with the use of the brightness estimates in AC: the brightest of the two candidates was chosen. This approach was applied only if the Tycho star was brighter than the Tycho completeness limit (otherwise the identification with the brightest candidate may be false due to the absence of the true counterpart star in the Tycho Catalogue), and if the brightness estimates of the $\mathrm{AC}$ images allowed a secure "bright"/ "faint" classification.

- "Two-to-two identification": two close Tycho stars matching two close AC images. Such cases were handled on the basis of the relative brightness of the candidates: the brightest of the two Tycho stars was identified with the brightest of the two AC images, and vice versa. Cases of this type were solved only if blue magnitudes of the Tycho stars and brightness estimates of the AC images differed enough to allow a secure "bright"/ "faint" classification.

\subsection{Identification results}

On the average, more than $98 \%$ of the Tycho stars satisfying the selection criteria (cf. Sect. 2.2.2) were identified in the AC. The identification percentage varied from zone to zone, between $96.43 \%$ (Cordoba) and $99.54 \%$ (Hyderabad South).

To conclude, we note that the identification of Tycho stars in the Astrographic Catalogue was based solely on constraining the upper limit of the proper motion modulus and on photometric data. It thus made no use of any further kinematic assumptions (e.g., choosing the closest candidate) to avoid kinematic biases in the TRC proper motions. 
Table 3. Estimated random and systematic accuracy of the preliminary TRC proper motions, for each AC zone. The individual columns denote: mean number of positions used to derive proper motions $\left(N_{\mathrm{P}}\right)$, rms internal standard error of the preliminary proper motions in units of mas/yr $\left(\hat{\sigma}\left(\mu_{\alpha *}\right)\right.$ and $\left.\hat{\sigma}\left(\mu_{\delta}\right)\right)$, residual magnitude and colour equation as estimated from the differences TRC(prelim)-Hipparcos (in units of mas $/ \mathrm{yr} / \mathrm{mag}$ ), and standard deviations of the preliminary TRC proper motions from Hipparcos $\left(\sigma\left(\Delta \mu_{\alpha *}\right)\right.$ and $\left.\sigma\left(\Delta \mu_{\delta}\right)\right)$

\begin{tabular}{lccccccccc}
\hline AC Zone & $N_{\mathrm{P}}$ & $\hat{\sigma}\left(\mu_{\alpha *}\right)$ & $\hat{\sigma}\left(\mu_{\delta}\right)$ & $\Delta \mu_{\alpha *}\left(V_{\mathrm{T}}\right)$ & $\Delta \mu_{\alpha *}\left(\left(B_{\mathrm{T}}-V_{\mathrm{T}}\right)\right)$ & $\Delta \mu_{\delta}\left(V_{\mathrm{T}}\right)$ & $\Delta \mu_{\delta}\left(\left(B_{\mathrm{T}}-V_{\mathrm{T}}\right)\right)$ & $\sigma\left(\Delta \mu_{\alpha *}\right)$ & $\sigma\left(\Delta \mu_{\delta}\right)$ \\
\hline Melbourne & 3.38 & 2.00 & 1.88 & +0.02 & +0.03 & -0.20 & -0.29 & 2.30 & 2.40 \\
Sydney & 3.33 & 3.06 & 2.78 & -0.11 & -0.41 & +0.10 & -0.39 & 3.54 & 3.53 \\
Cape & 3.00 & 2.89 & 2.73 & -0.35 & -0.21 & +0.03 & +0.22 & 3.17 & 3.12 \\
Perth & 3.14 & 2.51 & 2.47 & +0.42 & +0.45 & +0.30 & +0.25 & 3.05 & 2.95 \\
Cordoba & 2.84 & 2.85 & 2.58 & -0.25 & -0.17 & +0.00 & -0.11 & 3.44 & 3.16 \\
S.Hyderabad & 3.20 & 2.92 & 2.90 & -0.34 & +0.24 & +0.00 & +0.14 & 3.22 & 3.08 \\
Tacubaya & 3.05 & 2.12 & 2.03 & +0.51 & +0.28 & +0.15 & +0.41 & 2.56 & 2.48 \\
San Fernando & 2.98 & 2.44 & 2.30 & -0.21 & +0.04 & -0.08 & +0.28 & 2.61 & 2.46 \\
Algiers & 2.95 & 1.68 & 1.59 & -0.16 & -0.05 & +0.08 & +0.37 & 2.32 & 2.32 \\
Toulouse & 2.98 & 1.94 & 1.76 & +0.09 & +0.09 & +0.34 & +0.70 & 2.45 & 2.41 \\
Bordeaux & 3.03 & 1.72 & 1.56 & -0.32 & -0.37 & +0.01 & +0.35 & 2.34 & 2.21 \\
Paris & 3.16 & 1.62 & 1.47 & +0.06 & +0.04 & +0.15 & +0.50 & 2.03 & 2.07 \\
Oxford & 3.18 & 2.48 & 2.41 & -0.29 & -0.18 & -0.25 & +0.38 & 2.98 & 2.95 \\
Uccle & 2.57 & 4.59 & 4.53 & +0.23 & -0.13 & +0.20 & +0.71 & 4.68 & 4.61 \\
N.Hyderabad & 2.87 & 3.06 & 2.94 & -0.22 & -0.45 & +0.01 & -0.37 & 3.54 & 3.50 \\
Helsingfors & 3.08 & 1.71 & 1.66 & +0.01 & +0.14 & +0.32 & +0.56 & 2.00 & 2.10 \\
Catania & 3.31 & 2.24 & 2.14 & -0.10 & +0.21 & +0.12 & +0.13 & 2.59 & 2.60 \\
Vatican & 3.36 & 2.94 & 2.85 & -0.27 & -0.20 & +0.00 & -0.63 & 3.78 & 3.92 \\
Greenwich & 3.07 & 1.86 & 1.91 & -0.17 & -0.03 & -0.21 & -0.06 & 2.24 & 2.26 \\
Potsdam & 2.53 & 1.65 & 1.63 & +0.00 & +0.02 & +0.29 & +0.70 & 2.10 & 2.18 \\
\hline Mean values & 3.00 & 2.41 & 2.31 & 0.24 & 0.24 & 0.17 & 0.39 & 2.84 & 2.81 \\
\hline
\end{tabular}

\section{Derivation of proper motions}

The proper motion of a Tycho star was derived from its position at epoch J1991.25, as given in the Tycho Catalogue, and the AC observations identified with it. Although the typical number of AC observations per Tycho star is two, 20183 Tycho stars with only one observation in AC were also accepted for TRC.

\subsection{Least-squares adjustment}

The derivation of proper motions followed the standard weighted least-squares fit procedure, applied if more than two positions per star were available. The constraint on the upper limit of the Tycho proper motion modulus (cf. Sect. 4.2) allowed to use the simple linear model:

$$
\begin{aligned}
& \alpha\left(T_{i}\right)=\alpha\left(T_{0 \alpha}\right)+\mu_{\alpha}\left(T_{i}-T_{0 \alpha}\right), \\
& \delta\left(T_{i}\right)=\delta\left(T_{0 \delta}\right)+\mu_{\delta}\left(T_{i}-T_{0 \delta}\right),
\end{aligned}
$$

where the meaning of the various symbols is as follows: $T_{0 \alpha}, T_{0 \delta}$ - the weighted mean epochs of right ascension and declination; $\alpha\left(T_{0 \alpha}\right), \delta\left(T_{0 \delta}\right)$ - the weighted means of right ascension and declination at epoch $T_{0 \alpha}$ and $T_{0 \delta} ; T_{i}$, $\alpha\left(T_{i}\right), \delta\left(T_{i}\right)$ - epoch, right ascension, and declination of the individual observation; $\mu_{\alpha}$ and $\mu_{\delta}$ - resulting proper motion components in right ascension and declination.
The individual positions were weighted by the inverse square of their respective standard errors. Those of the Tycho positions were defined by the published standard errors of right ascension and declination, while those of the $\mathrm{AC}$ positions were estimated from the rms scatter of the plate reduction residuals (the same for all measurements on a given plate) and estimates for the systematic errors of the individual positions, based on the covariance matrix of the plate parameters derived at step 3 of the reduction procedure (see Sect. 3). The Tycho Catalogue data is treated as one observation per star in this context.

The chi-square of the residuals after each least-squares adjustment was compared with the expected value. Any large discrepancy indicates a problem case due to misidentification, unresolved duplicity etc. Stars with one of the chi-squares (for $\alpha$ or $\delta$ ) exceeding a certain limit were rejected. The rate of such rejections was $1.31 \%$.

The rms internal estimates of the errors of the preliminary proper motion components, $\hat{\sigma}\left(\mu_{\alpha *}\right)$ and $\hat{\sigma}\left(\mu_{\delta}\right)$, derived from the covariance matrices of Eqs. (6), are given in Table 3. The estimates are provided separately for each zone to emphasize the effects of the zonal structure of the Astrographic Catalogue, e.g. the impact of the smaller epoch difference to the Tycho data in the AC zones covering the original Potsdam zone. The rms all-sky $\hat{\sigma}\left(\mu_{\alpha *}\right)$ and $\hat{\sigma}\left(\mu_{\delta}\right)$ values for the 990182 TRC stars are 2.37 and $2.27 \mathrm{mas} / \mathrm{yr}$ respectively. These are slightly different from 
the corresponding values listed in the last row of Table 3 due to the zonal overlaps, i.e. the one-degree declination strips on the border of any two AC zones, covered by both of them.

\subsection{Investigation of the systematic errors of the preliminary proper motions}

In order to investigate the reliability of the internal error estimates, the preliminary TRC proper motions were compared with those from the Hipparcos Catalogue. The comparison was based on 93078 stars common to the two catalogues. The distribution of the differences is shown in Fig. 8; the standard deviations are 2.73 mas/yr in $\mu_{\alpha *}$ and $2.69 \mathrm{mas} / \mathrm{yr}$ in $\mu_{\delta}$. Comparing the latter with the rms value of the internal error estimates $(2.37 / 2.27 \mathrm{mas} / \mathrm{yr})$ and taking into account rms standard error of the Hipparcos proper motions (1.25 mas/yr per component), we conclude that the preliminary TRC proper motions include residual systematic errors of the order of $1 \mathrm{mas} / \mathrm{yr}$.

Probable sources of the residual systematic errors are suggested by the nature of the internal error estimates. These represent only the random errors of the individual positions and a part of the residual plate-scale systematic errors. Errors not covered by the internal estimates include:

- errors of the reference system realization at the AC epoch (caused by the residual systematic errors of the improved ACRS used as an intermediate reference catalogue for the $\mathrm{AC}$ reduction), and

- residual magnitude and colour equations in the $\mathrm{AC}$ data.

Zonal analysis of the preliminary TRC-Hipparcos differences confirmed that the systematic errors are mainly caused by the residual magnitude equation in the $\mathrm{AC}$ data. Estimates of the residual magnitude and colour equations in the preliminary TRC proper motions, derived by linear interpolation from the comparison with Hipparcos, are
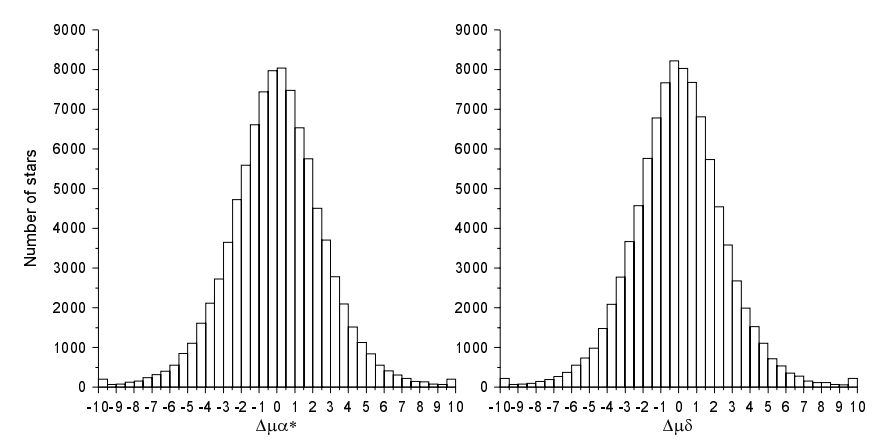

Fig. 8. Differences of the preliminary TRC and Hipparcos proper motions, in units of mas/yr, based on 93078 common stars listed in Table 3 ; on the average, the errors amount to $0.25 \mathrm{mas} / \mathrm{yr} / \mathrm{mag}$ each.

It should be noted that the residual magnitude and colour equations found in the preliminary TRC proper motions are not independent. This is explained by the strong correlation between colour and magnitude of the Tycho Catalogue stars: for example, if stars common to TRC and Hipparcos are divided according to the median $\left(B_{\mathrm{T}}-V_{\mathrm{T}}\right)$ colour index (0.64 mag) into a "blue" and a "red" subset, the median magnitude is $B_{\mathrm{T}}=8.8 \mathrm{mag}$ for the "blue", and $B_{\mathrm{T}}=9.8 \mathrm{mag}$ for the "red" one. Additional investigation had shown magnitude equation to be the dominant effect, and the colour equation was therefore ignored in the derivation of further systematic corrections.

\subsection{Correction of the zonal magnitude equation}

As mentioned above (cf. Sect. 3.7), the plate adjustment approach used to reduce the AC onto the Hipparcos system cannot completely remove the magnitude equation, primarily due to the insufficient density of the reference catalogue. For the same reason we cannot derive completely adequate a-posteriori correction of the residual magnitude equation found in the preliminary TRC proper motions. To account at least for the major part of the effect, declination-dependent linear corrections were derived from comparison with Hipparcos.

In order to derive the corrections, the differences between the preliminary TRC and the Hipparcos proper motions for common stars were organized into a table of $\left(\delta, \Delta \mu_{\alpha *}, \Delta \mu_{\delta}\right)$ and sorted according to increasing declination. Batches of 500 successive rows of this table with 50 per cent overlap were then used. In each batch, the stars were divided according to $B_{\mathrm{T}}$ in two equal subsets, and the median values of the magnitude and of the differences in proper motion components were calculated for each subset. The magnitude equation was then assumed to be linear through the two points, but was truncated for magnitudes brighter than $B_{\mathrm{T}}=6.5 \mathrm{mag}$ to avoid large, unwarranted corrections for very bright stars. For each batch the linear magnitude equation estimate was calculated in the form of the intercept at $B_{\mathrm{T}}=9.35 \mathrm{mag}$ (in mas/yr) and the slope (in mas/yr/mag). These corrections were then applied to the preliminary TRC to yield the final proper motions.

\section{Proper motions quality assessment}

To derive truly external error estimates of the final TRC proper motions would require an independent set of proper motions of comparable (or better) quality. The only catalogue satisfying the quality requirement - the Hipparcos Catalogue - was used for the AC reduction. It is therefore unsuitable for the purpose, due to the correlation with 

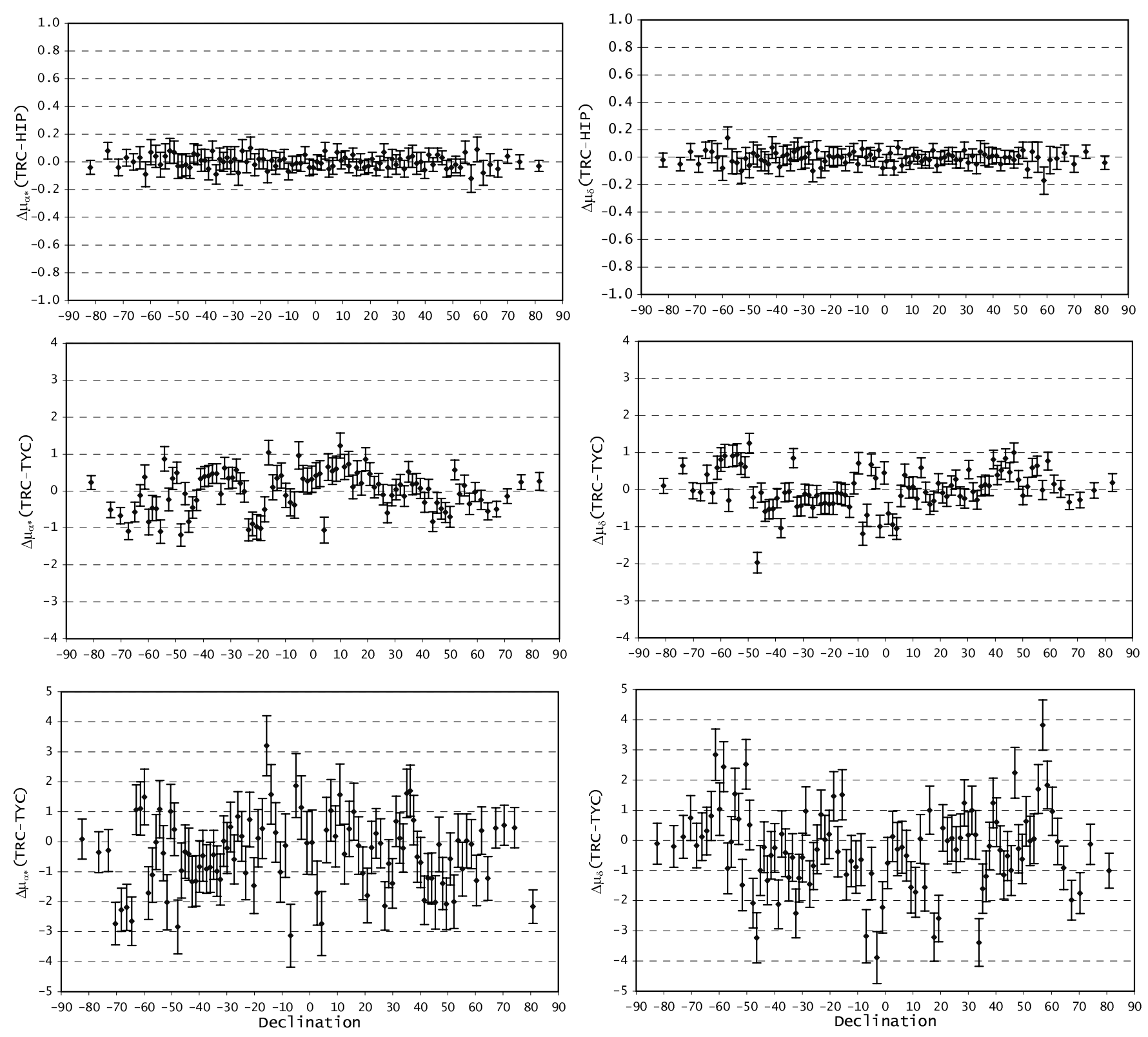

Fig. 9. Systematic differences between the final TRC proper motions and the proper motions in the Hipparcos and Tycho Catalogues, in units of mas/yr, as functions of declination. Top row: proper motion differences TRC-Hipparcos for 93078 stars of median magnitude $B_{\mathrm{T}}=9.35 \mathrm{mag}$; each point represents the mean of 1000 differences. Middle row: TRC-Tycho for 458601 stars brighter than $B_{\mathrm{T}}=11.25 \mathrm{mag}$; each point represents the mean of 5000 differences. Bottom row: TRC-Tycho for 491636 stars fainter than $B_{\mathrm{T}}=11.25 \mathrm{mag}$

TRC. Under such circumstances, the only reasonable approach is to estimate the external errors as a combination of the internal standard errors and estimated residual systematic errors.

The internal error estimates (cf. Sect. 5.1) assess the random errors of the proper motions, plus a portion of the residual plate-scale systematic errors. The rms value of the internal estimates is $2.3 \mathrm{mas} / \mathrm{yr}$ per proper motion component.

The comparison of the final TRC proper motions with Hipparcos (Fig. 9, top row) suggests that for bright (median $B_{\mathrm{T}}=9.35 \mathrm{mag}$ ) stars the residual systematic errors do not exceed 0.2 mas/yr. But, of course, this estimate will be too optimistic due to the correlation between TRC and Hipparcos.

Another resource for the estimation of the residual TRC systematic errors, especially for faint stars, is the Tycho Catalogue. Tycho proper motions, though of poor precision, are in the nature of the case tied to the Hipparcos system and should be virtually free from magnitude equation. Unfortunately, the Tycho proper motions have been shown to possess systematic errors 
depending on ecliptic latitude, with values reaching $1 \mathrm{mas} / \mathrm{yr}$ for some latitudes (Høg et al. 1998a). This fact, which actually prevented a direct application of the Tycho proper motions to the correction of the zonal magnitude equation, should be taken into account in the following comparison.

The comparison of the TRC and Tycho proper motions for stars brighter than $B_{\mathrm{T}}=11.25 \mathrm{mag}$ (Fig. 9, middle row) shows that the systematic errors of TRC at this magnitude are less than 0.5 mas/yr rms. For fainter stars (Fig. 9, bottom row) the comparison suggests that a considerable magnitude equation is still present in the TRC proper motions. Based on the comparison, the residual systematic errors of the TRC proper motions for stars fainter than $B_{\mathrm{T}}=11.25$ mag may be estimated as $1 \mathrm{mas} / \mathrm{yr} \mathrm{rms}$.

Summing up, we estimate the external errors of the final TRC proper motions as 2.4 mas/yr per component, by a combination of the internal error estimates $(2.3 \mathrm{mas} / \mathrm{yr}$ $\mathrm{rms}$ ) and estimated residual systematic errors (0.5 mas/yr rms at $\left.B_{\mathrm{T}}=11.25 \mathrm{mag}\right)$. Nonetheless, we should note the possibility of larger residual systematic errors (up to $2 \mathrm{mas} / \mathrm{yr}$ ) for the faintest TRC stars in some declination zones.

\section{Catalogue content}

The Tycho Reference Catalogue contains 990182 stars. For each of them, the following data is provided:

- Tycho identifier.

- Right ascension and declination at epoch J2000.0 with respect to the ICRS reference system. The equatorial coordinates given in the catalogue were derived from the right ascensions and declinations at the respective mean epochs (cf. Sect. 5.1) by applying the TRC proper motions. From the comparison with Hipparcos, the accuracy of the TRC positions at epoch J2000.0 is estimated as $25-45$ mas, depending on magnitude. This is close to the Tycho positional accuracy at epoch J1991.25, as expected.

- Standard errors of right ascension and declination for the respective mean epochs.

- Proper motion components in right ascension and declination, along with their estimated external errors. The catalogue provides final proper motions; the error estimates given in the catalogue represent a combination of the internal standard errors and the residual systematic error estimates derived from comparing TRC with the Hipparcos and Tycho Catalogues (cf. Sect. 6).

- Tycho photometry and the respective standard errors.

- Additional data related to the proper motion derivation, e.g. the mean epoch of right ascensions and declinations, the number of positions used to derive the proper motion, and proper motion quality flag.
- Flags indicating known or suspected variability/duplicity.

- Cross-identification with Hipparcos and other catalogues.

A detailed description of the catalogue can be found in the documentation accompanying the machine-readable version of the Tycho Reference Catalogue, made available at the Centre de Données Astronomiques de Strasbourg (CDS).

\section{TRC \& ACT}

In parallel with the TRC production, the US Naval Observatory undertook a similar project based on the proprietary machine-readable version of the AC (Urban et al. 1998a). The resulting "Astrographic Catalogue and Tycho" (ACT) Catalogue (Urban et al. 1998b) has a lot in common with TRC:

- Both catalogues have the same aim, viz. to extend the ICRS/Hipparcos system to fainter stars.

- The two catalogues are based on exactly the same observational material: the Astrographic Catalogue and the Tycho Catalogue.

- Both groups had to solve exactly the same set of problems: AC reduction onto the Hipparcos system, identification of Tycho stars in AC, etc.

This means that TRC and ACT might be expected to be of very similar stellar content and quality. A first comparison (Høg et al. 1998a) roughly confirmed this expectation. However, this does by no means say that the two catalogues are essentially identical: there are lots of differences due to the different catalogue construction strategies. We illustrate this by just two examples.

The basic difference between the identification techniques used by TRC and ACT is that the latter adopted the proper motions given in the Tycho Catalogue. Prior to identification, the position of a Tycho star was propagated to the AC plate epoch using the published proper motion. This difference led to discrepant identifications in ACT and TRC and, in consequence, to large disagreements of the proper motion of some stars in the two catalogues.

Concerning the $\mathrm{AC}$ reduction there is a major difference in the sequence of the individual steps, which influenced the results for all stars. For TRC, the intermediate reference catalogue (ACRS) was first transformed to the ICRS/Hipparcos system, and then used for the derivation of the zone-specific corrections to the AC data. The Hipparcos Catalogue served as a reference at the final reduction step (cf. Sect. 3.1) to put individual AC plates onto the ICRS system. For ACT, the AC reduction was done on the original ACRS, and the resulting AC positions were subsequently transformed to the Hipparcos system by means of a filtering approach (Urban et al. 1998a). 
There is also an important difference in the meaning of the published data that any potential user of either catalogue should be aware of. While the standard errors given in $\mathrm{ACT}$ (1.6 - $1.8 \mathrm{mas} / \mathrm{yr}$ per proper motion component) represent the internal errors only, the corresponding TRC data attempt to incorporate at least some of the remaining systematic errors. For bright stars, the standard deviation of the ACT-Hipparcos differences is 3.5-3.8 mas/yr (Urban et al. 1998b).

\section{Conclusions}

The Tycho Reference Catalogue is a new reference catalogue which provides an accurate, dense and stable realization of the ICRS reference system in the optical domain. TRC realizes the ICRS system at epoch J2000.0 with a median accuracy of 45 mas. Its high-precision proper motions, derived using the hundred years old Astrographic Catalogue data, ensure a reference system realization with an accuracy better than 100 mas over the time span of several decades. The quality of the TRC proper motions demonstrates the immense potential of the Astrographic Catalogue for the massive derivation of high-precision proper motions. It should be noted that TRC, although containing a million stars, still utilizes less than a quarter of the AC data.

Further development of the Tycho Reference Catalogue is planned. Reprocessing of the Tycho data, now under way at Copenhagen University Observatory (Høg et al. 1998b), will result in the Tycho-2 Catalogue containing positions and photometry of 2500000 stars, scheduled to appear in 1999. Proper motions will also be included, based on a conventional adjustment of the Astrographic Catalogue onto the ICRS/Hipparcos system, like the present versions of TRC and ACT.

A major improvement of the systematic accuracy of the TRC proper motions can be expected in another version, to be called TRC2, which will be based on the Tycho2 Catalogue positions and photometry and a global block adjustment of the Astrographic Catalogue, undertaken at Sternberg Astronomical Institute. Reduction of the AC using plate overlap technique will enable:

- direct reduction of the Astrographic Catalogue onto the Hipparcos system, eliminating the need for an intermediate reference catalogue;

- simultaneous and correct (from the statistical point of view) determination of the significant plate-scale systematic errors based on all plates, without the partial averaging that was used to yield the zone-specific corrections under the present approach;

- detailed investigation of the magnitude and colour equations, réseau and micrometer errors, and other types of systematic errors probably present in the AC data which were neglected under the classical approach.
Acknowledgements. The TRC would not have been possible without its two main foundations, the machine-readable Astrographic Catalogue and the Tycho Catalogue. The former resulted from many years of work by the Sternberg Astronomical Institute's Astrometry Division, headed by Vilen Nesterov. The latter marks the great success of the Tycho Data Analysis Consortium under the leadership of Erik Høg. The time and effort by the project leaders, and many other individuals who contributed to the projects, to ensure the highest possible quality of the catalogues is gratefully acknowledged. The third indispensable ingredient to TRC was the primary optical reference frame provided by the Hipparcos Catalogue.

The TRC project was supported by the Russian Foundation for Basic Research, by the Danish Space Board and the Velux Foundation of 1981, by the Swedish National Space Board and by the Deutsche Forschungsgemeinschaft.

We finally acknowledge constructive comments of the referee, J. Kovalevsky, that helped to clarify the presentation.

\section{References}

Appenzeller I., 1998, Transactions of the IAU 23B

Arias E.F., Charlot P., Feissel M., Lestrade J.-F., 1995, A\&A 303, 604

Baldwin J.M., 1926, Melbourne Astrographic Catalogue 1900.0. Vol. I, H.J. Green Gov. Printer, Melbourne

Bastian U., Röser S., Yagudin L.I., Nesterov V.V., Polozhentsev D.D., Potter Kh.I., Wielen R., Yatskiv Ya.S., 1993, PPM Star Catalogue. Positions and proper motions of 197179 stars south of $-2.5^{\circ}$ declination for equinox and epoch J2000.0. Vols. III, IV. Spektrum Akademischer Verlag, Heidelberg

Bergeron J., 1992, Transactions of the IAU 21B, p. 41

Corbin T.E., Urban S.E., 1991, Astrographic Catalogue Reference Stars. U.S. Naval Observatory, Washington

Debarbat S., Eddy J.A., Eichhorn H.K., Upgren A.R., 1988, IAU Symposium 133, "Mapping the Sky - Past Heritage and Future Directions", Paris, France, June 1-5, 1987. Kluwer Academic Publishers, Dordrecht

Eichhorn H.K., 1960, Astron. Nachr. 285, 233

Eichhorn H.K., 1974, Astronomy of Star Positions. Frederick Ungar, New York

Eichhorn H.K., Gatewood G.D., 1966, AJ 72, 1191

ESA 1997, The Hipparcos and Tycho Catalogues, ESA SP-1200

Gulyaev A.P., Nesterov V.V., 1992, On the four million stars catalogue. Moscow University Press, Moscow, in Russian

Gureeva E.Yu., 1992, in: Gulyaev \& Nesterov 1992, p. 43

Høg E., Kuzmin A., Bastian U., Fabricius C., Kuimov K., Lindegren L., Makarov V.V., Röser S., 1998a, A\&A 335, L65

Høg E., Fabricius C., Makarov V.V., Bastian U., Schwekendiek P., Wicenec A., 1998b, in: International colloquium "Modern Astrometry and Astrodynamics" honouring H. Eichhorn, Vienna, Austria, May 25-26, 1998. Kluwer Academic Publishers, Dordrecht (in press)

Hudson D.J., 1964, Lectures on Statistics and Probability. CERN, Geneva

Jenkner H., Lasker B.M., Sturch C.R., McLean B.J., Shara M.M., Russell J.L., 1990, AJ 99, 2082 
Kovalevsky J., Lindegren L., Perryman M.A.C., et al., 1997, A\&A 323, 620

Kuimov K.V., 1992, in: Gulyaev \& Nesterov 1992, p. 27

Kuimov K.V, Kuzmin A.V., Nesterov V.V., 1998, BRAS Phys. 62,1774

Kuzmin A., Gulyaev A., Nesterov V., Kuimov K., Sementsov V., Bastian U, Röser S., 1997a, In: IAU Symposium 179 "New Horizons from Multi-Wavelength Sky Surveys", Baltimore, U.S.A., August 26-31, 1996; McLean B., Golombek D., Hayes J., Payne H. (eds.). Kluwer Academic Publishers, Dordrecht, p. 409

Kuzmin A., Bastian U., Høg E., Kuimov K., Röser S., 1997b, in: ESA Symposium "Hipparcos - Venice'97", Venice, Italy, 13-16 May, 1997; ESA SP-402, p. 125

Lasker B.M., Sturch C.R., McLean B.J., Russell J.L., Jenkner H., Shara M.M., 1990, AJ 99, 2019

Lindegren L., Röser S., Schrijver H., et al., 1995, A\&A 304, 44

Luyten W.J., 1979, New Luyten Catalogue of Stars with Proper Motions Larger than Two Tenths of an Arcsecond. University of Minnesota, Minneapolis

Luyten W.J., Hughes H.S., 1980, Proper Motion Survey with the Forty-Eight Inch Schmidt Telescope. LV. First Supplement to the NLTT Catalogue. University of Minnesota, Minneapolis

Ma C., Arias E.F., Eubanks T.M., Fey A.L., Gontier A.-M., Jacobs C.S., Sovers O.J., Archinal B.A., Charlot P., 1998, AJ 116, 516
Ma C., Feissel M., 1997, IERS Technical Note \# 23, Observatoire de Paris

Nesterov V.V., Kisljuk V.S., Potter H.I., 1991, in: IAU Symposium 141 "Inertial Coordinate System on the Sky", Leningrad, USSR, October 17-21, 1989; Lieske J.H., Abalakin V.K. (eds.). Kluwer Academic Publishers, Dordrecht, p. 482

Pocock R.J., 1918, Astrographic Catalogue 1900.0, Hyderabad Section, Vol. I. Neill \& Co., Edinburgh

Röser S., Bastian U., 1991, PPM Star Catalogue, Positions and proper motions of 181731 stars north of $-2.5^{\circ}$ declination for equinox and epoch J2000.0, Vols. I, II. Spektrum Akademischer Verlag, Heidelberg

Röser S., Høg E., 1993, in: Workshop on databases for Galactic structure, Swarthmore, PA, U.S.A., May 17-19, 1993; Philip A.G.D., Hauck B., Upgren A.R. (eds.). L. Davis Press, Schenectady, p. 137

Russell J.L., Lasker B.M., McLean B.J., Sturch C.R., Jenkner H., 1990, AJ 99, 2059

Trepied M.Ch., 1903, Catalogue Photographique du Ciel 1900, Observatoire d'Alger, Vol. I. Gauthier-Villars, Paris

Urban S.E., Corbin T.E., Wycoff G.L., et al., 1998a, AJ 115, 1212

Urban S.E., Corbin T.E., Wycoff G.L., Martin J.C., Jackson E.S., Zacharias M.I., Hall D.M., 1998b, AJ 115, 2161 